\title{
Some conditions for the oscillation of second- order differential equations with several mixed delays
}

\author{
Shyam Sundar Santra® and Andrea Scapellato®
}

\begin{abstract}
In this work, we obtain necessary and sufficient conditions for the oscillation of the solutions to a second-order neutral differential equation with mixed delays. Two examples are provided to show effectiveness and feasibility of main results. Our main tool is the Lebesgue's Dominated Convergence theorem.
\end{abstract}

Mathematics Subject Classification. 34C10, 34K11.

Keywords. Oscillation, delay, neutral, Lebesgue's dominated convergence theorem.

\section{Introduction}

In this paper, we are interested in the study of certain oscillation properties of second-order differential equations containing mixed several delays.

Nowadays, the study of qualitative properties of ordinary differential equations attracts considerable attention from the scientific community due to numerous applications of them to several contexts, such as Biology, Physics, Chemistry, and Dynamical Systems. For some details related to the recent studies on oscillation and non-oscillation properties, exponential stability, instability, existence of unbounded solutions of the equations under consideration, we refer the reader to the books $[1,2]$.

It is worthy pointing out that both oscillation and stability criteria are currently used in the studies of non-linear mathematical models with delay for single species and several species with interactions, in logistic models, $\alpha$-delay models, mathematical models with varying capacity, mathematical models for food-limited population dynamics with periodic coefficients, and diffusive logistic models (for instance, diffusive Malthus-type models with several delays, autonomous diffusive delayed logistic models with Neumann boundary conditions, periodic diffusive logistic Volterra-type models with delays, and so on). 
The literature is full of very interesting results related to the oscillation properties for second-order differential equations. Now, we recall some studies that possess a strong connection with the content of this paper. In [3], Baculíková, Li, and Džurina obtained some oscillation criteria for the following second-order neutral differential equations:

$$
\left(p(t)[u(t)+q(t) u(\varsigma(t))]^{\prime}\right)^{\prime}+r(t) u(\sigma(t))+v(t) u(\eta(t))=0
$$

considering the cases in which the arguments are delayed, advanced, or mixed.

In [4], Arul and Shobha investigated some oscillation properties of the solutions of the following equation:

$$
\left(p(t) z^{\prime}(t)\right)^{\prime}+r(t) u(\sigma(t))=0, \quad t \geq t_{0} \geq 0,
$$

being $z(t)=u(t)+a(t) u(t-\tau)+b(t) u(t+\delta)$.

In [5], Thandapani and Rama considered the second-order non-linear neutral differential equations of mixed type. Precisely, the authors studied the following neutral differential equations:

$$
\begin{aligned}
& {\left[\left(u(t)+a u\left(t-\varsigma_{1}\right)-b u\left(t+\varsigma_{2}\right)\right)^{\alpha}\right]^{\prime \prime}=q(t) u^{\beta}\left(t-\sigma_{1}\right)+p(t) u^{\beta}\left(t+\sigma_{2}\right),} \\
& {\left[\left(u(t)-a u\left(t-\varsigma_{1}\right)+b u\left(t+\varsigma_{2}\right)\right)^{\alpha}\right]^{\prime \prime}=q(t) u^{\beta}\left(t-\sigma_{1}\right)+p(t) u^{\beta}\left(t+\sigma_{2}\right),}
\end{aligned}
$$

being $\alpha$ and $\beta \geq 1$ defined as the ratios of odd positive integers. Some generalizations of the results discussed in [5] are contained in [6].

More general results are contained in [7] where Thandapani, Padmavathi, and Pinelas derived oscillation theorems for even-order non-linear neutral differential equations with mixed type with the following form:

$$
\left(p(t)\left(u(t)+b u\left(t-\varsigma_{1}\right)+c u\left(t+\varsigma_{2}\right)\right)^{(n-1)}\right)^{\prime}+r(t) u^{\alpha}\left(t-\sigma_{1}\right)+q(t) u^{\beta}\left(t+\sigma_{2}\right)=0
$$

being $t \geq t_{0}, n \geq 2$ an even integer, $\alpha \geq 1$ and $\beta \geq 1$ ratios of odd positive integers. The case in which $n$ is odd was treated for slightly different equations in $[8,9]$.

It is interesting to notice that in the aforementioned works, the authors obtained only sufficient conditions that ensure the oscillation of the solutions of the considered equations. A problem worthy of investigations is the study of necessary and sufficient conditions for the oscillation and some satisfactory answers were given in $[10,13]$.

Finally, we refer the interested reader to the following paper and to the references therein for some recent results on the oscillation theory for ordinary differential equations of several orders [11-18].

In this work, we deal with necessary and sufficient conditions for the oscillation of solutions to a second-order non-linear differential equations of the form

$$
\left(p(t)\left(w^{\prime}(t)\right)^{\alpha}\right)^{\prime}+\sum_{j=1}^{m} r_{j}(t) g_{j}\left(u\left(\nu_{j}(t)\right)\right)=0, \quad t \geq t_{0}
$$

where

$$
w(t)=u(t)+q(t) u(\varsigma(t))
$$


being the functions $g_{j}, r_{j}, p, q, \nu_{j}, \varsigma$ continuous and such that the following conditions stated below hold:

(a) $\nu_{j} \in C([0, \infty), \mathbb{R}), \varsigma \in C^{2}([0, \infty), \mathbb{R})$, if we consider the simple delay, then $\nu_{j}(t)<t, \varsigma(t)<t, \lim _{t \rightarrow \infty} \nu_{j}(t)=\infty, \lim _{t \rightarrow \infty} \varsigma(t)=\infty$;

(b) $\nu_{j} \in C([0, \infty), \mathbb{R}), \varsigma \in C^{2}([0, \infty), \mathbb{R})$, if we consider the advanced delay, then (a) can be modified by $\nu_{j}(t)>t, \varsigma(t)<t, \lim _{t \rightarrow \infty} \nu_{j}(t)=\infty$, $\lim _{t \rightarrow \infty} \varsigma(t)=\infty$;

(c) $p \in C^{1}([0, \infty), \mathbb{R}), r_{j} \in C([0, \infty), \mathbb{R}) ; 0<p(t), 0 \leq r_{j}(t)$ for all $t \geq 0$ and $j=1,2, \ldots, m ; \sum r_{j}(t)$ is not identically zero in any interval $[b, \infty)$;

(d) $q \in C^{2}\left([0, \infty), \mathbb{R}_{+}\right)$with $0 \leq q(t) \leq a<1$;

(e) $g_{j} \in C(\mathbb{R}, \mathbb{R})$ is non-decreasing and $g_{j}(t) t>0$ for $t \neq 0, j=1,2, \ldots, m$;

(f) $\lim _{t \rightarrow \infty} P(t)=\infty$ where $P(t)=\int_{0}^{t} p^{-1 / \alpha}(s) \mathrm{d} s$;

(g) $\alpha$ is the quotient of two positive odd integers.

For the sake of completeness, we recall some basic definitions. A solution of (1.1) is a function $u:\left[t_{u}, \infty\left[\rightarrow \mathbb{R}, t_{u} \geq t_{0}\right.\right.$, such that $p(t)\left(w^{\prime}(t)\right)^{\alpha}$ and $u(t)$ are continuously differentiable for all $t \in\left[t_{u}, \infty[\right.$ and it satisfies (1.1) for all $t \in\left[t_{u}, \infty[\right.$. A solution $u(t)$ of (1.1) is said to be non-oscillatory if it is eventually positive or eventually negative; otherwise, it is said to be oscillatory. Finally, we say that (1.1) is oscillatory if all of its solutions are oscillatory.

\section{Preliminary results}

For the sake of simplicity, we set

$$
R_{1}(t)=\sum_{j=1}^{m} r_{j}(t) g_{j}\left((1-a) w\left(\nu_{j}(t)\right)\right) .
$$

Lemma 2.1. Suppose that (a)-(g) hold for $t \geq t_{0}$ and that $u$ is an eventually positive solution of (1.1). Then, $w$ satisfies

$$
0<w(t), \quad w^{\prime}(t)>0, \quad \text { and } \quad\left(p(t)\left(w^{\prime}(t)\right)^{\alpha}\right)^{\prime} \leq 0 \quad \text { for } t \geq t_{1} .
$$

Proof. Let $u$ be an eventually positive solution. Then, $w(t)>0$ and there exists $t_{0} \geq 0$, such that $u(t)>0, u\left(\nu_{j}(t)\right)>0, u(\varsigma(t))>0$ for all $t \geq t_{0}$ and $j=1,2, \ldots, m$. Then, (1.1) gives that

$$
\left(p(t)\left(w^{\prime}(t)\right)^{\alpha}\right)^{\prime}=-\sum_{j=1}^{m} r_{j}(t) g_{j}\left(u\left(\nu_{j}(t)\right)\right) \leq 0,
$$

which shows that $p(t)\left(w^{\prime}(t)\right)^{\alpha}$ is non-increasing for $t \geq t_{0}$. Next, we claim that $p(t)\left(w^{\prime}(t)\right)^{\alpha}$ is positive for $t \geq t_{1}>t_{0}$. If not, there exists a point $t \geq t_{1}>t_{0}$, such that $p(t)\left(w^{\prime}(t)\right)^{\alpha} \leq 0$. Therefore, we can choose $c>0$, such that

$$
p(t)\left(w^{\prime}(t)\right)^{\alpha} \leq-c
$$

that is

$$
w^{\prime}(t) \leq(-c)^{1 / \alpha} p^{-1 / \alpha}(t)
$$


Integrating both sides from $t_{1}$ to $t$, we get

$$
w(t)-w\left(t_{1}\right) \leq(-c)^{1 / \alpha}\left(P(t)-P\left(t_{1}\right)\right) .
$$

Taking the limit of both sides as $t \rightarrow \infty$, we have $\lim _{t \rightarrow \infty} w(t) \leq-\infty$ which leads to a contradiction to $w(t)>0$. Hence, $p(t)\left(w^{\prime}(t)\right)^{\alpha}>0$ for $t \geq t_{1}$, that is, $w^{\prime}(t)>0$ for $t \geq t_{1}$. This completes the proof.

Lemma 2.2. Suppose that (a)-(g) hold for $t \geq t_{0}$ and that $u$ is an eventually positive solution of (1.1). Then, $w$ satisfies

$$
u(t) \geq(1-a) w(t) \quad \text { for } t \geq t_{1} .
$$

Proof. Assume that $u$ be an eventually positive solution of (1.1). Then, $w(t)>0$ and there exists $t \geq t_{1}>t_{0}$, such that

$$
\begin{aligned}
u(t) & =w(t)-q(t) u(\varsigma(t)) \\
& \geq w(t)-q(t) w(\varsigma(t)) \\
& \geq w(t)-q(t) w(t) \\
& =(1-q(t)) w(t) \\
& \geq(1-a) w(t) .
\end{aligned}
$$

Hence, $w$ satisfies (2.3) for $t \geq t_{1}$.

\section{Main results}

In Theorem 3.1, we use a constant $\beta$, quotient of two odd positive integers with $\beta>\alpha$, for which

$$
\frac{g_{j}(t)}{t^{\beta}} \text { is non-decreasing for } 0<t, j=1,2, \ldots, m .
$$

Existence of such constant can be established by taking $g_{j}(t)=|y|^{\delta} \operatorname{sgn}(t)$, with $\beta<\delta$.

Theorem 3.1. Let (b)-(g) and (3.1) for $t \geq t_{0}$. Then, every solution of (1.1) is oscillatory if and only if

$$
\int_{0}^{\infty} p^{-1 / \alpha}(s)\left[\int_{s}^{\infty} \sum_{j=1}^{m} r_{j}(\psi) \mathrm{d} \psi\right]^{1 / \alpha} \mathrm{d} s=\infty .
$$

Proof. Let $u$ is an eventually positive solution of (1.1). Then, $w(t)>0$ and there exists $t_{0} \geq 0$, such that $u(t)>0, u\left(\nu_{j}(t)\right)>0, u(\varsigma(t))>0$ for all $t \geq t_{0}$ and $j=1,2, \ldots, m$. Thus, Lemmas 2.1 and 2.2 hold for $t \geq t_{1}$. By Lemma 2.1 , there exists $t_{2}>t_{1}$, such that $w^{\prime}(t)>0$ for all $t \geq t_{2}$. Then, there exist $t_{3}>t_{2}$ and $c>0$, such that $w(t) \geq c$ for all $t \geq t_{3}$. Next, using Lemma 2.2, we wet $u(t) \geq(1-a) w(t)$ for all $t \geq t_{3}$ and (1.1) become

$$
\left(p(t)\left(w^{\prime}(t)\right)^{\alpha}\right)^{\prime}+R_{1}(t) \leq 0 \text { for } y \neq \phi_{k} \text {. }
$$


Integrating (3.3) from $t$ to $\infty$, we get

$$
\left[p(s)\left(w^{\prime}(s)\right)^{\alpha}\right]_{t}^{\infty}+\int_{t}^{\infty} R_{1}(s) \mathrm{d} s \leq 0 .
$$

Since $p(t)\left(w^{\prime}(t)\right)^{\alpha}$ is positive and non-decreasing $\lim _{t \rightarrow \infty} p(t)\left(w^{\prime}(t)\right)^{\alpha}$ finitely exists and positive

that is

$$
p(t)\left(w^{\prime}(t)\right)^{\alpha} \geq \int_{t}^{\infty} R_{1}(s) \mathrm{d} s
$$

Since

$$
w^{\prime}(t) \geq p^{-1 / \alpha}(t)\left[\int_{t}^{\infty} R_{1}(s) \mathrm{d} s\right]^{1 / \alpha}
$$

$$
\begin{aligned}
g_{j}\left[(1-a) w\left(\nu_{j}(t)\right)\right] & =\frac{g_{j}\left[(1-a) w\left(\nu_{j}(t)\right)\right]}{(1-a)^{\beta} w^{\beta}\left(\nu_{j}(t)\right)}(1-a)^{\beta} w^{\beta}\left(\nu_{j}(t)\right) \\
& \geq \frac{g_{j}[c(1-a)]}{c^{\beta}(1-a)^{\beta}}(1-a)^{\beta} w^{\beta}\left(\nu_{j}(t)\right) \\
& =\frac{g_{j}[c(1-a)]}{c^{\beta}} w^{\beta}\left(\nu_{j}(t)\right) .
\end{aligned}
$$

Then, we use (3.5) in (3.4) to get

$$
w^{\prime}(t) \geq p^{-1 / \alpha}(t)\left[\int_{t}^{\infty} \sum_{j=1}^{m} r_{j}(s) \frac{g_{j}[c(1-a)]}{c^{\beta}} w^{\beta}\left(\nu_{j}(s)\right) \mathrm{d} s\right]^{1 / \alpha} .
$$

Next, if we set $K=\frac{g_{0}[c(1-a)]}{c^{\beta}}$ where $g_{0}[c(1-a)]=\min _{1 \leq j \leq m} g_{j}[c(1-a)]$, the above inequality becomes

$$
w^{\prime}(t) \geq K^{1 / \alpha} p^{-1 / \alpha}(t)\left[\int_{t}^{\infty} \sum_{j=1}^{m} r_{j}(s) w^{\beta}\left(\nu_{j}(s)\right) \mathrm{d} s\right]^{1 / \alpha} .
$$

Using (b) and $w(t)$ is non-decreasing, we have

$$
w^{\prime}(t) \geq K^{1 / \alpha} p^{-1 / \alpha}(t)\left[\int_{t}^{\infty} \sum_{j=1}^{m} r_{j}(s) \mathrm{d} s\right]^{1 / \alpha} w^{\beta / \alpha}(t),
$$

that is

$$
\frac{w^{\prime}(t)}{w^{\beta / \alpha}(t)} \geq K^{1 / \alpha} p^{-1 / \alpha}(t)\left[\int_{t}^{\infty} \sum_{j=1}^{m} r_{j}(s) \mathrm{d} s\right]^{1 / \alpha}
$$

Integrating both sides from $t_{3}$ to $\infty$, we get

$$
K^{1 / \alpha} \int_{t_{3}}^{\infty} p^{-1 / \alpha}(s)\left[\int_{s}^{\infty} \sum_{j=1}^{m} r_{j}(\psi) \mathrm{d} \psi\right]^{1 / \alpha} \mathrm{d} s \leq \int_{t_{3}}^{\infty} \frac{w^{\prime}(s)}{w^{\beta / \alpha}(s)} \mathrm{d} s<\infty
$$

due to $\beta>\alpha$, which is a contradiction to (3.2), and hence, the sufficiency part of the theorem is proved. 
Next, we prove necessary part reasoning by contradiction. If (3.2) does not hold, then for every $\varepsilon>0$, there exists $t \geq t_{0}$ for which

$$
\int_{t}^{\infty} p^{-1 / \alpha}(s)\left[\int_{s}^{\infty} \sum_{j=1}^{m} r_{j}(\psi) \mathrm{d} \psi\right]^{1 / \alpha} \mathrm{d} s<\varepsilon \quad \text { for } t \geq Y
$$

where $2 \varepsilon=\left[\max _{\{1 \leq j \leq m\}} g_{j}\left(\frac{1}{1-a}\right)\right]^{-1 / \alpha}>0$.

Let us define the set

$$
V=\left\{u \in C([0, \infty)): \frac{1}{2} \leq u(t) \leq \frac{1}{1-a} \text { for all } t \geq Y\right\}
$$

and $\Phi: V \rightarrow V$ as

$$
(\Phi u)(t)= \begin{cases}0 & \text { if } t \leq Y, \\ \frac{1+a}{2(1-a)}-q(t) u(\varsigma(t)) & \\ +\int_{T}^{t} p^{-1 / \alpha}(s)\left[\int_{s}^{\infty} \sum_{j=1}^{m} r_{j}(\psi) g_{j}\left(u\left(\nu_{j}(\psi)\right)\right) \mathrm{d} \psi\right]^{1 / \alpha} \mathrm{d} s & \text { if } t>Y .\end{cases}
$$

Now we prove that $(\Phi u)(t) \in V$. For $u(t) \in V$, we have

$$
\begin{aligned}
(\Phi u)(t) & \leq \frac{1+a}{2(1-a)}+\int_{T}^{t} p^{-1 / \alpha}(s)\left[\int_{s}^{\infty} \sum_{j=1}^{m} r_{j}(\psi) g_{j}\left(\frac{1}{1-a}\right) \mathrm{d} \psi\right]^{1 / \alpha} \mathrm{d} s \\
& \leq \frac{1+a}{2(1-a)}+\left[\max _{1 \leq j \leq m} g_{j}\left(\frac{1}{1-a}\right)\right]^{1 / \alpha} \cdot \varepsilon \\
& =\frac{1+a}{2(1-a)}+\frac{1}{2}=\frac{1}{1-a}
\end{aligned}
$$

and furthermore, for $u(t) \in V$,

$$
(\Phi u)(t) \geq \frac{1+a}{2(1-a)}-q(t) \cdot \frac{1}{1-a}+0 \geq \frac{1+a}{2(1-a)}-\frac{a}{1-a}=\frac{1}{2} .
$$

Hence, $\Phi$ maps from $V$ to $V$. Now, we find a fixed point for $\Phi$ in $V$ which will give an eventually positive solution of (1.1). To this end, we define a sequence of functions in $V$ by

$$
\begin{array}{r}
u_{0}(t)=0 \text { for } t \geq_{0}, \\
u_{1}(t)=\left(\Phi u_{0}\right)(t)=\left\{\begin{array}{ll}
0 & \text { if } t<Y \\
\frac{1}{2} & \text { if } t \geq Y
\end{array},\right. \\
u_{n+1}(t)=\left(\Phi u_{n}\right)(t) \text { for } n \geq 1, t \geq Y .
\end{array}
$$

We have $u_{1}(t) \geq u_{0}(t)$ for each fixed $t$ and $\frac{1}{2} \leq u_{n-1}(t) \leq u_{n}(t) \leq \frac{1}{1-a} \quad t \geq$ $Y$ for all $n \geq 1$. Thus, $\left(u_{n}\right)_{n \in \mathbb{N}}$ converges pointwise to a function $u$. By Lebesgue's Dominated Convergence Theorem, $u$ is a fixed point of $\Phi$ in $V$, which shows that there is a non-oscillatory solution. This completes the proof of the theorem. 
In Theorem 3.2, we use a constant $\beta$, quotient of two odd positive integers with $\beta<\alpha$, for which

$$
\frac{g_{j}(t)}{t^{\beta}} \text { is non-increasing for } 0<t, j=1,2, \ldots, m \text {. }
$$

Existence of such constant can be established by taking $g_{j}(t)=|y|^{\delta} \operatorname{sgn}(t)$, with $\beta>\delta$. The assumption upon $\beta$ can be withdrawn by taking $|u|^{\beta} \operatorname{sgn}(u)$ instead of $u^{\beta}$.

Theorem 3.2. Let (a), (c)-(g) and (3.6) hold for $t \geq t_{0}$. Then, every solution of (1.1) is oscillatory if

$$
\frac{1}{(2 c)^{\beta}}\left[\int_{0}^{\infty} \sum_{j=1}^{m} r_{j}(\psi) g_{j}\left[c(1-a) P\left(\nu_{j}(\psi)\right)\right] \mathrm{d} \psi\right]=\infty \quad \forall c \neq 0 .
$$

Proof. Let $u(t)$ be an eventually positive solution of (1.1). Then, proceeding as in Theorem 3.1, we have $t_{2}>t_{1}>t_{0}$, such that Eq. (3.4) holds for all $t \geq t_{2}$. Using (e), there exists $t_{3}>t_{2}$ for which $P(t)-P\left(t_{3}\right) \geq \frac{1}{2} P(t)$ for $t \geq t_{3}$. Integrating (3.4) from $t_{3}$ to $t$, we have

$$
\begin{aligned}
w(t)-w\left(t_{3}\right) & \geq \int_{t_{3}}^{t} p^{-1 / \alpha}(s)\left[\int_{s}^{\infty} R_{1}(\kappa) \mathrm{d} \kappa\right]^{1 / \alpha} \mathrm{d} s \\
& \geq \int_{t_{3}}^{t} p^{-1 / \alpha}(s)\left[\int_{t}^{\infty} R_{1}(\kappa) \mathrm{d} \kappa\right]^{1 / \alpha} \mathrm{d} s,
\end{aligned}
$$

that is

$$
\begin{aligned}
w(t) & \geq\left(P(t)-P\left(t_{3}\right)\right)\left[\int_{t}^{\infty} R_{1}(\kappa) \mathrm{d} \kappa\right]^{1 / \alpha} \\
& \geq \frac{1}{2} P(t)\left[\int_{t}^{\infty} R_{1}(\kappa) \mathrm{d} \kappa\right]^{1 / \alpha} .
\end{aligned}
$$

Since $p(t)\left(w^{\prime}(t)\right)^{\alpha}$ is non-increasing and positive, then there exists $c>0$ and $t_{4}>t_{3}$, such that $p(t)\left(w^{\prime}(t)\right)^{\alpha} \leq c^{\alpha}$ for $t \geq t_{4}$. Integrating the relation $w^{\prime}(t) \leq c p^{-1 / \alpha}(t)$ from $t_{4}$ to $t$, we have

$$
w(t)-w\left(t_{4}\right) \leq c\left(P(t)-P\left(t_{4}\right)\right)
$$

that is

$$
w(t) \leq c P(t) \quad \text { for } t \geq t_{4} .
$$

Using (3.6) and (3.9), we obtain

$$
\begin{aligned}
g_{j}\left[(1-a) w\left(\nu_{j}(t)\right)\right] & =\frac{g_{j}\left[(1-a) w\left(\nu_{j}(t)\right)\right]}{(1-a)^{\beta} w^{\beta}\left(\nu_{j}(t)\right)}(1-a)^{\beta} w^{\beta}\left(\nu_{j}(t)\right) \\
& \geq \frac{g_{j}\left[c(1-a) P\left(\nu_{j}(t)\right)\right]}{c^{\beta}(1-a)^{\beta} P^{\beta}\left(\nu_{j}(t)\right)}(1-a)^{\beta} w^{\beta}\left(\nu_{j}(t)\right) \\
& =\frac{g_{j}\left[c(1-a) P\left(\nu_{j}(t)\right)\right]}{c^{\beta} P^{\beta}\left(\nu_{j}(t)\right)} w^{\beta}\left(\nu_{j}(t)\right) \quad \forall t \geq t_{4} .
\end{aligned}
$$


Using (3.10) in (3.8), we obtain

$$
w(t) \geq \frac{1}{2} P(t)\left[\int_{t}^{\infty} \sum_{j=1}^{m} r_{j}(\kappa) \frac{g_{j}\left[c(1-a) P\left(\nu_{j}(\kappa)\right)\right]}{c^{\beta} P^{\beta}\left(\nu_{j}(\kappa)\right)} w^{\beta}\left(\nu_{j}(\kappa)\right) \mathrm{d} \kappa\right]^{1 / \alpha} .
$$

Hence

$$
w(t) \geq \frac{1}{2} P(t) U^{1 / \alpha}(t) \quad \text { for } t \geq t_{4},
$$

where

$$
U(t)=\frac{1}{c^{\beta}}\left[\int_{t}^{\infty} \sum_{j=1}^{m} r_{j}(\kappa) g_{j}\left[c(1-a) P\left(\nu_{j}(\kappa)\right)\right] \frac{w^{\beta}\left(\nu_{j}(\kappa)\right)}{P^{\beta}\left(\nu_{j}(\kappa)\right)} \mathrm{d} \kappa\right] .
$$

Now

$$
\begin{aligned}
U^{\prime}(t)= & -\frac{1}{c^{\beta}} \sum_{j=1}^{m} r_{j}(t) g_{j}\left[c(1-a) P\left(\nu_{j}(t)\right)\right] \frac{w^{\beta}\left(\nu_{j}(t)\right)}{P^{\beta}\left(\nu_{j}(t)\right)} \\
& \leq-\frac{1}{(2 c)^{\beta}} \sum_{j=1}^{m} r_{j}(t) g_{j}\left[c(1-a) P\left(\nu_{j}(t)\right)\right] U^{\beta / \alpha}\left(\nu_{j}(t)\right) \leq 0,
\end{aligned}
$$

which shows that $U(t)$ is non-increasing on $\left[t_{4}, \infty\right)$ and $\lim _{t \rightarrow \infty} U(t)$ exists. Using (3.11) and (a), we find

$$
\begin{aligned}
{\left[U^{1-\beta / \alpha}(t)\right]^{\prime} } & =(1-\beta / \alpha) U^{-\beta / \alpha}(t) U^{\prime}(t) \\
& \leq-\frac{1-\beta / \alpha}{(2 c)^{\beta}} \sum_{j=1}^{m} r_{j}(t) g_{j}\left[c(1-a) P\left(\nu_{j}(t)\right)\right] U^{\beta / \alpha}\left(\nu_{j}(t)\right) U^{-\beta / \alpha}(t) \\
& \leq-\frac{1-\beta / \alpha}{(2 c)^{\beta}} \sum_{j=1}^{m} r_{j}(t) g_{j}\left[c(1-a) P\left(\nu_{j}(t)\right)\right] .
\end{aligned}
$$

Now, integrating (3.13) from $t_{4}$ to $t$, we have

$$
\left[U^{1-\beta / \alpha}(s)\right]_{t_{4}}^{t} \leq-\frac{1-\beta / \alpha}{(2 c)^{\beta}} \int_{t_{4}}^{t} \sum_{j=1}^{m} r_{j}(s) g_{j}\left[c(1-a) P\left(\nu_{j}(s)\right)\right] \mathrm{d} s,
$$

that is

$$
\frac{1-\beta / \alpha}{(2 c)^{\beta}} \int_{0}^{\infty} \sum_{j=1}^{m} r_{j}(s) g_{j}\left[c(1-a) P\left(\nu_{j}(s)\right)\right] \mathrm{d} s \leq-\left[U^{1-\beta / \alpha}(s)\right]_{t_{4}}^{t}<U^{1-\beta / \alpha}\left(t_{4}\right)<\infty,
$$

which contradicts (3.7). This completes the proof.

Example 3.1. Consider the neutral differential equation

$$
\left(\left(\left(u(t)+\mathrm{e}^{-t} u(\varsigma(t))\right)^{\prime}\right)^{1 / 3}\right)^{\prime}+t(u(t-2))^{7 / 3}+(t+1)(u(t-3))^{11 / 3}=0 .
$$

Here, $\alpha=1 / 3, p(t)=1,0<q(t)=\mathrm{e}^{-t}<1 \nu_{j}(t)=t-(j+1), g_{j}(t)=$ $t^{(4 j+3) / 3}$. For $\beta=5 / 3$, we have $\delta_{j}=(4 j+3) / 3>\beta=5 / 3>\alpha=1 / 3$, and 
$g_{1}(t) / t^{\beta}=t^{2 / 3}$ and $g_{2}(t) / t^{\beta}=t^{2}$ which are both increasing functions. Now, we check (3.2). We have

$$
\begin{aligned}
& \int_{t_{0}}^{\infty}\left[\frac{1}{p(s)}\left[\int_{s}^{\infty} \sum_{j=1}^{m} r_{j}(\psi) d \psi\right]\right]^{1 / \alpha} d s \\
& \geq \int_{t_{0}}^{\infty}\left[\frac{1}{p(s)}\left[\int_{s}^{\infty} r_{1}(\psi) d \psi\right]\right]^{1 / \alpha} d s \\
& \geq \int_{2}^{\infty}\left[\int_{s}^{\infty} \psi d \psi\right]^{3} d s=\infty .
\end{aligned}
$$

Therefore, all the conditions of of Theorem 3.1 hold. Thus, each solution of (3.14) is oscillatory.

Example 3.2. Consider the neutral differential equation

$$
\left(\mathrm{e}^{-t}\left(\left(u(t)+\mathrm{e}^{-t} u(\varsigma(t))\right)^{\prime}\right)^{11 / 3}\right)^{\prime}+\frac{1}{t+1}(u(t-2))^{1 / 3}+\frac{1}{t+2}(u(t-3))^{5 / 3}=0 .
$$

Here, $\alpha=11 / 3, p(t)=\mathrm{e}^{-t}, 0<q(t)=\mathrm{e}^{-t}<1, \nu_{j}(t)=t-(j+1)$, $P(t)=\int_{0}^{t} \mathrm{e}^{3 s / 11} d s=\frac{11}{3}\left(\mathrm{e}^{3 t / 11}-1\right), g_{j}(t)=t^{(4 j-3) / 3}$. For $\beta=7 / 3$, we have $\delta_{j}=(4 j-3) / 3<\beta=7 / 3<\alpha=11 / 3$, and $g_{1}(t) / t^{\beta}=t^{-2}$ and $g_{2}(t) / t^{\beta}=t^{-2 / 3}$ which are both decreasing functions. Now, we check (3.7).

We have

$$
\begin{aligned}
& \frac{1}{(2 c)^{\beta}}\left[\int_{0}^{\infty} \sum_{j=1}^{m} r_{j}(\psi) g_{j}\left[c(1-a) P\left(\nu_{j}(\psi)\right)\right] d \psi\right] \\
& \geq \frac{1}{(2 c)^{7 / 3}} \int_{0}^{\infty} r_{1}(\psi) g_{1}\left[c(1-a) P\left(\nu_{1}(\psi)\right)\right] \mathrm{d} \psi \\
& =\frac{1}{(2 c)^{7 / 3}} \int_{0}^{\infty} \frac{1}{\psi+1}\left[c(1-a) \frac{11}{3}\left(\mathrm{e}^{3(\psi-2) / 11}-1\right)\right]^{1 / 3} d \psi=\infty \quad \forall c>0 .
\end{aligned}
$$

Therefore, all the conditions of Theorem 3.2 hold, and therefore, each solution of (3.15) is oscillatory.

\section{Conclusions}

In this work, we considered the second-order highly non-linear neutral differential equations with several mixed delays (1.1) and established necessary and sufficient conditions for the oscillation of (1.1) when the neutral coefficient $q$ lies in $[0,1)$.

Funding Open access funding provided by Universitá degli Studi di Catania within the CRUI-CARE Agreement. 
Open Access. This article is licensed under a Creative Commons Attribution 4.0 International License, which permits use, sharing, adaptation, distribution and reproduction in any medium or format, as long as you give appropriate credit to the original author(s) and the source, provide a link to the Creative Commons licence, and indicate if changes were made. The images or other third party material in this article are included in the article's Creative Commons licence, unless indicated otherwise in a credit line to the material. If material is not included in the article's Creative Commons licence and your intended use is not permitted by statutory regulation or exceeds the permitted use, you will need to obtain permission directly from the copyright holder. To view a copy of this licence, visit http:// creativecommons.org/licenses/by/4.0/.

Publisher's Note Springer Nature remains neutral with regard to jurisdictional claims in published maps and institutional affiliations.

\section{References}

[1] Berezansky, L., Domoshnitsky, A., Koplatadze, R.: Oscillation, Nonoscillation, Stability and Asymptotic Properties for Second and Higher Order Functional Differential Equations. CRC Pres, Boca Raton (2020)

[2] Agarwal, R.P., O'Regan, D., Saker, S.H.: Oscillation and Stability of Delay Models in Biology. Springer, New York (2014)

[3] Baculíková, B., Li, T., Džurina, J.: Oscillation theorems for second order neutral differential equations. Electron. J. Qual. Theory Differ. Equ. 74, 1-13 (2011)

[4] Arul, R., Shobha, V.S.: Oscillation of second order neutral differential equations with mixed neutral term. Int. J. Pure Appl. Math. 104(2), 181-191 (2015)

[5] Thandapani, E., Rama, R.: Oscillation criteria for second-order nonlinear neutral differential equations of mixed type. Electron. J. Qual. Theory Differ. Equ. 75, 1-16 (2012)

[6] Thandapani, E., Selvarangam, S., Vijaya, M., Rama, R.: Oscillation results for second order nonlinear differential equation with delay and advanced arguments. Kyungpook Math. J. 56, 137-146 (2016)

[7] Thandapani, E., Padmavathi, S., Pinelas, S.: Oscillation criteria for even-order nonlinear neutral differential equations of mixed type. Bull. Math. Anal. Appl. 6(1), 9-22 (2014)

[8] Li, T., Thandapani, E.: Oscillation of solutions to odd-order nonlinear neutral functional differential equations. Electron. J. Differ. Equ. 2011(23), 1-12 (2011)

[9] Thandapani, E., Padmavathy, S., Pinelas, S.: Oscillation criteria for odd-order nonlinear differential equations with advanced and delayed arguments. Electron. J. Differ. Equ. 2014(174), 1-13 (2014)

[10] Pinelas, S., Santra, S.S.: Necessary and sufficient condition for oscillation of nonlinear neutral first-order differential equations with several delays. J. Fixed Point Theory Appl. 20, Article number: 27 (2018)

[11] Santra, S.S., Ghosh, T., Bazighifan, O.: Explicit criteria for the oscillation of second-order differential equations with several sub-linear neutral coefficients. Adv. Differ. Equ. 2020, 643 (2020) 
[12] Santra, S.S., Dassios, I., Ghosh, T.: On the asymptotic behavior of a class of second-order non-linear neutral differential equations with multiple delays. Axioms 9, 134 (2020)

[13] Santra, S.S.: Necessary and sufficient conditions for oscillation of second-order delay differential equations. Tatra Mt. Math. Publ. 75, 135-146 (2020)

[14] Öcalan, O., Kili, N., Özkan, U.: Oscillatory behavior of nonlinear advanced differential equations with a non-monotone argument. Acta Math. Univ. Comenianae 88(2), 239-246 (2019)

[15] Li, T., Rogovchenko, Y.V.: Oscillation theorems for second-order nonlinear neutral delay differential equations. Abstr. Appl. Anal. 2014(ID 594190), 1-11 (2014)

[16] Li, T., Rogovchenko, Y.V.: Oscillation of second-order neutral differential equations. Math. Nachr. 288, 1150-1162 (2015)

[17] Li, Q., Wang, R., Chen, F., Li, T.: Oscillation of second-order nonlinear delay differential equations with nonpositive neutral coefficients. Adv. Differ. Equ. 20, 7 (2015)

[18] Li, T., Rogovchenko, Y.V.: Oscillation criteria for second-order superlinear Emden-Fowler neutral differential equations. Monatsh. Math. 184, 489-500 (2017)

\author{
Shyam Sundar Santra \\ Department of Mathematics \\ JIS College of Engineering \\ Kalyani 741235 \\ India \\ e-mail: shyam01.math@gmail.com \\ Andrea Scapellato \\ Dipartimento di Matematica e Informatica \\ Università degli Studi di Catania \\ Viale Andrea Doria 6 \\ 95125 Catania \\ Italy \\ e-mail: andrea.scapellato@unict.it
}

Accepted: December 9, 2021. 